\title{
THE MILNOR SIGNATURES OF COMPOUND KNOTS
}

\author{
C. KEARTON
}

ABSTRACT. The Milnor signatures of a classical knot are related to those of its companions.

1. We shall work throughout in the smooth or piecewise-linear category. A $k n o t k$ is a circle $S^{1}$ embedded in the 3-sphere $S^{3}$. A regular neighbourhood $V$ of $k$ is a solid torus. A longitude of $\partial V$ is a circle embedded in $\partial V$ which is homologous to $k$ in $V$, and null-homologous in the closed complement of $V$. We assume that all knots and longitudes are oriented.

Let $T$ be a solid torus unknotted in $S^{3}$ and containing a knot $l^{*}$, and let $f$ be a faithful map from $T$ onto $V$ (that is, a homeomorphism which takes a longitude of $\partial T$ onto a longitude of $\partial V$ ). If $l^{*}$ represents $n \in \mathbf{Z}=H_{1}(T)$, then $l=f\left(l^{*}\right)$ is homologous to $n k$ in $V$. Proofs will be presented as though $n$ were positive, but with trivial adjustments in notation they are valid for all $n$.

Let $\Delta_{l}(t)$ be the Alexander polynomial of the knot $l$, and $\Delta_{k}(t), \Delta_{l^{*}}(t)$ those of $k, l^{*}$ respectively. It is a result of Seifert [S] that $\Delta_{l}(t)=\Delta_{k}\left(t^{n}\right) \cdot \Delta_{l^{*}}(t)$.

If $p(t)$ is a symmetric, quadratic factor of $\Delta_{k}(t)$, irreducible over the real numbers, then we can write $p(t)$ in the form $t^{-1}-2 \cos \theta+t, 0<\theta<\pi$. Milnor $\left[\mathbf{M}_{1}\right]$ has defined a signature $\sigma_{\theta}(k)$ which is an invariant of $k$. Let $p\left(t^{n}\right)=p_{1}(t) \cdots p_{n}(t)$, where each $p_{r}(t)$ is symmetric, quadratic and irreducible over the real numbers, and let $\exp \left(i \theta_{r}\right)$ be the root of $p_{r}(t)$ which is also an $n$th root of $\exp (i \theta)$, where $0<\left|\theta_{r}\right|<\pi$.

THEOREM. $\sigma_{\left|\theta_{r}\right|}(l)=\sigma_{\left|\theta_{r}\right|}\left(l^{*}\right)+\sigma_{\theta}(k) \operatorname{sign}\left(n \sin \theta_{r}\right)$, where $\sigma_{\left|\theta_{r}\right|}\left(l^{*}\right)=0$ if $\exp \left(i \theta_{r}\right)$ is not a root of $\Delta_{l *}(t)$.

If $\exp (i \varphi)$ is a root of $\Delta_{l^{*}}(t)$ but not of $\Delta_{k}\left(t^{n}\right)$, then $\sigma_{\varphi}(l)=\sigma_{\varphi}\left(l^{*}\right)$.

Corollary.

$$
\begin{aligned}
\sigma(l) & =\sigma\left(l^{*}\right) \text { if } n \text { is even, } \\
& =\sigma\left(l^{*}\right)+\sigma(k) \text { if } n \text { is odd. }
\end{aligned}
$$

Here $\sigma(k)$ denotes the signature of $k$, and is just the sum over all $\theta$ of $\sigma_{\theta}(k)$. The latter result is due to Shinohara [Sh].

2. Let $K$ be the closed complement in $S^{3}$ of the solid torus $V$, and let $\tilde{K}$ be the infinite cyclic cover of $K$ corresponding to the kernel of the Hurewicz

Received by the editors April 24, 1978.

AMS (MOS) subject classifications (1970). Primary 55A25; Secondary 55A10, $55 C 05$.

Key words and phrases. Knot, Milnor signature. 
map $\pi_{1}(K) \rightarrow H_{1}(K) \cong(t:)$. Then $H_{1}(\tilde{K})$ is a finitely-generated module over $\Lambda=\mathbf{Z}\left[t, t^{-1}\right]$, and there is a Blanchfield duality pairing

$$
\langle,\rangle: H_{1}(\tilde{K}) \times H_{1}(\tilde{K}) \rightarrow \Lambda_{0} / \Lambda,
$$

where $\Lambda_{0}$ is the field of fractions of $\Lambda$. This pairing is Hermitian with respect to the conjugation defined by $t \mapsto t^{-1}$. It is also nonsingular.

Set $\Gamma=\mathbf{R}\left[t, t^{-1}\right]$, and pass to real coefficients: then we obtain a pairing

$$
\langle,\rangle: H_{1}(\tilde{K} ; \mathbf{R}) \times H_{1}(\tilde{K} ; \mathbf{R}) \rightarrow \Gamma_{0} / \Gamma .
$$

Let $p(t)$ be a prime in $\Gamma$ dividing $\Delta_{k}(t)$; and let $V_{p}$ denote the $p(t)$-primary component of $H_{1}(\tilde{K} ; \mathbf{R})$. As in $[\mathbf{K}], V_{p}$ is orthogonal to $V_{q}$ unless $(p(t))=$ $\left(q\left(t^{-1}\right)\right)$. Moreover, if $p(t)=p\left(t^{-1}\right)$, then $V_{p}$ can be written as an orthogonal direct sum $V_{p}^{1} \oplus \cdots \oplus V_{p}^{m}$, with $V_{p}^{r}$ a free module over $\Gamma /\left(p^{r}\right)$. Let $(x)$ denote the image in $H_{p}^{r}=V_{p}^{r} / p V_{p}^{r}$ of $x$ in $V_{p}^{r}$; if $x, y \in V_{p}^{r}$, then we can define $[(x),(y)]_{p}^{r}=\left\langle p(t)^{r-1} x, y\right\rangle$.

Let $\varphi: \Gamma \rightarrow \Gamma /(p)$ be the quotient map; then defining $((x),(y))_{p}^{r}$ to be $\varphi(z)$, where $[(x),(y)]_{p}^{r}=z / p$, makes $H_{p}^{r}$ into an Hermitian space over the field $\Gamma /(p) \cong \mathbf{C}$. Conjugation coincides with complex conjugation, as the roots of $p(t)$ lie on the complex unit circle. Let $\sigma_{p}^{r}(k)$ be the signature of the corresponding quadratic space, and let $\sigma_{p}(k)$ be the sum over odd $r$ of the $\sigma_{p}^{r}(k)$.

It is shown in $[\mathbf{K}]$ that $\sigma_{p}(k)=\sigma_{\theta}(k)$, where $p(t)=t^{-1}-2 \cos \theta+t$, $0<\theta<\pi$.

In passing, note that $\sigma_{p}(k)$ is an invariant of the cobordism class of $k$ (see $\left.\left[\mathbf{M}_{1}\right]\right) ; \sigma_{p}^{r}(k)$ is an invariant of $k$, but not of its cobordism class [L]. As Milnor points out $\left[\mathbf{M}_{2}\right]$, for $r$ even the corresponding quadratic space is hyperbolic, and so $\sigma_{\theta}^{r}(k)=0$.

3. Let $N$ be a regular neighbourhood in $T$ of $l^{*}, L^{*}$ the closed complement of $N$ in $S^{3}, L^{\prime}$ the closed complement of $f(N)$ in $V$, and $L$ the closed complement of $f(N)$ in $S^{3}$. Then $L=L^{\prime} \cup K$, and $L^{\prime} \cap K=\partial V$. Passing to the infinite cyclic cover of $L, \tilde{L}=\tilde{L}^{\prime} \cup \tilde{K}_{1} \cup \cdots \cup \tilde{K}_{n}$, where the $\tilde{K}_{r}$ are disjoint copies of $\tilde{K}$, and $\tilde{L}^{\prime} \cap \tilde{K}_{r} \cong S^{1} \times \mathbf{R}$. We can number the $\tilde{K}_{r}$ so that the action of $(t:)$ on $\tilde{L}$ is given by $t \tilde{K}_{r}=\tilde{K}_{r+1}$, working modulo $n$.

It is implicit in the work of Seifert [S] that $H_{1}(\tilde{L})$ splits as a direct sum of $\Lambda$-modules, $H_{1}\left(\tilde{L}^{*}\right) \oplus H_{1}\left(\tilde{K}_{1} \cup \cdots \cup \tilde{K}_{n}\right)$. Furthermore, if $M(t)$ is a presentation matrix for $H_{1}(\tilde{K})$, then $M\left(t^{n}\right)$ is a presentation matrix for $H_{1}\left(\tilde{K}_{1}\right.$ $\left.\cup \cdots \cup \tilde{K}_{n}\right)$ : this too is easily deduced from [S; p. 32].

From the definition of the Blanchfield duality pairing [B], it is clear that the direct sum above is orthogonal, and that $H_{1}\left(\tilde{K}_{1} \cup \cdots \cup \tilde{K}_{n}\right)$ splits as an orthogonal direct sum of Z-modules $H_{1}\left(\tilde{K}_{1}\right) \oplus \cdots \oplus H_{1}\left(\tilde{K}_{n}\right)$.

4. Let $p(t)=t^{-1}-2 \cos \theta+t, 0<\theta<\pi$, be an irreducible factor of $\Delta_{k}(t)$, and let $p\left(t^{n}\right)=p_{1}(t) \cdots p_{n}(t)$ where $p_{r}(t)=t^{-1}-2 \cos \theta_{r}+t, 0<$ $\left|\theta_{r}\right|<\pi$. Let $\tau=\exp (i \theta)$, and let $\tau_{r}=\exp \left(i \theta_{r}\right)$ be the root of $p_{r}(t)$ which is also an $n$th root of $\tau$. Write $p^{r}(t)=p\left(t^{n}\right) / p_{r}(t)$. 
Recall that $V_{p}$ is the $p(t)$-primary component of $H_{1}(\tilde{K} ; \mathbf{R})$. If we identify $H_{1}(\tilde{K} ; \mathbf{R})$ with $H_{1}\left(\tilde{K}_{1} ; \mathbf{R}\right) \subset H_{1}\left(\tilde{K}_{1} \cup \cdots \cup \tilde{K}_{n} ; \mathbf{R}\right)$, as a vector space, then clearly $\left(p^{r}(t)\right)^{N} V_{p}$ is contained in $V_{p_{r}}$, the $p_{r}(t)$-primary component of $H_{1}\left(\tilde{K}_{1}\right.$ $\left.\cup \cdots \cup \tilde{K}_{n} ; \mathbf{R}\right)$, for large $N$. Indeed, by considering a diagonal presentation matrix $M(t)$ for $H_{1}(\tilde{K} ; \mathbf{R})$, and passing to $M\left(t^{n}\right)$, it is clear that $\left(p^{r}(t)\right)^{N} V_{p}=$ $V_{p_{r}}$.

Consider $x, y \in V_{p}$ as elements of $H_{1}(\tilde{K} ; \mathbf{R})$; then $\langle x, y\rangle=\mu(t) /(p(t))^{m}$ say. Regarding $x, y$ as elements of $H_{1}\left(\tilde{K}_{1} \cup \cdots \cup \tilde{K}_{n} ; \mathbf{R}\right)$, it follows from the definition of the duality pairing that $\langle x, y\rangle=\mu\left(t^{n}\right) /\left(p\left(t^{n}\right)\right)^{m}$. Thus if $V_{p}=$ $V_{p}^{1} \oplus \cdots \oplus V_{p}^{m}$, an orthogonal direct sum in $H_{1}(\tilde{K} ; \mathbf{R})$, we can take $V_{p_{r}}^{s}=$ $\left(p^{r}(t)\right)^{N} V_{p}^{s}$ to obtain an orthogonal direct sum $V_{p_{r}}=V_{p_{r}}^{1} \oplus \cdots \oplus V_{p_{r}}^{m}$.

Let $x^{\prime}, y^{\prime} \in H_{p_{r}}^{s}$, and choose $x, y \in V_{p}^{s}$ so that $x^{\prime}=\left(\left(p^{r}(t)\right)^{N} x\right), y^{\prime}=$ $\left(\left(p^{r}(t)\right)^{N} y\right)$. Then

$$
\begin{aligned}
{\left[x^{\prime}, y^{\prime}\right]_{p_{r}}^{s} } & =\left\langle p_{r}(t)^{s-1}\left(p^{r}(t)\right)^{N} x,\left(p^{r}(t)\right)^{N} y\right\rangle \\
& =\left(p^{r}(t)\right)^{2 N-s+1}\left\langle p\left(t^{n}\right)^{s-1} x, y\right\rangle \\
& =\left(p^{r}(t)\right)^{2 N-s+1} \mu\left(t^{n}\right) / p\left(t^{n}\right)
\end{aligned}
$$

where regarding $x, y$ as elements of $H_{1}(\tilde{K} ; \mathbf{R})$, the Blanchfield pairing of $k$ gives $\left\langle p(t)^{s-1} x, y\right\rangle=\mu(t) / p(t)$. Thus

$$
\left[x^{\prime}, y^{\prime}\right]_{p_{r}}^{s}=\left(p^{r}(t)\right)^{2 N-s} \mu\left(t^{n}\right) / p_{r}(t)
$$

and so

$$
\left(x^{\prime}, y^{\prime}\right)_{p_{r}}^{s}=\left(p^{r}\left(\tau_{r}\right)\right)^{2 N-s} \mu(\tau) .
$$

Of course, if we regard $x, y$ as elements of $H_{1}(\tilde{K} ; \mathbf{R})$, then in the Hermitian space $H_{p}^{s}$ we have $((x),(y))_{p}^{s}=\mu(\tau)$. Thus it only remains for us to evaluate $p^{r}\left(\tau_{r}\right)$. Using L'Hôpital's rule, it is easy to see that

$$
p^{r}\left(\tau_{r}\right)=\lim _{t \rightarrow \tau_{r}}\left(\frac{p\left(t^{n}\right)}{p_{r}(t)}\right)=n \frac{\mathcal{G}(\tau)}{\mathcal{G}\left(\tau_{r}\right)}=n \frac{\sin \theta}{\sin \theta_{r}},
$$

where $\mathcal{G}(z)$ is the imaginary part of $z$.

Thus if $s$ is odd, $H_{p_{r}}^{s}$ contributes $\sigma_{\theta}^{s}(k) \operatorname{sign}\left(n \sin \theta_{r}\right)$ to the signature of $l$; and if $s$ is even, all the corresponding signatures are zero. This proves the theorem.

The corollary follows easily by considering the distribution of the $\tau_{r}$ around the unit circle.

\section{REFERENCES}

[B] R. C. Blanchfield, Intersection theory of manifolds with operators with applications to knot theory, Ann. of Math. 65 (1957), 340-356.

[K] C. Kearton, Signatures of knots and the free differential calculus, Quart J. Math. (to appear). 
[L] J. Levine, Invariants of knot cobordism, Invent. Math. 8 (1969), 98-110.

$\left[\mathrm{M}_{1}\right]$ J. Milnor, Infinite cyclic coverings, J. G. Hocking, Ed., Conf. on the Topology of Manifolds, Prindle, Weber and Schmidt, Boston, Mass., 1968, pp. 115-133.

$\left[\mathrm{M}_{2}\right]$, On isometries of inner product spaces, Invent. Math. 8 (1969), 83-97.

[S] H. Seifert, On the homology invariants of knots, Quart. J. Math. Oxford Ser. (2) 1 (1950), 23-32.

[Sh] Y. Shinohara, On the signature of knots and links, Trans. Amer. Math. Soc. 156 (1971), 273-285.

College of St. Hitd and St. Bede, Durham, DH1 1SZ, England 